\title{
CORRECTION OF BLEPHAROCONJUNCTIVITIS- RELATED UPPER EYELID ENTROPION USING THE ANTERIOR LAMELLAR REPOSITION TECHNIQUE
}

\author{
M. C. RHATIGAN, J. L. ASHWORTH, K. GOODALL and B. LEATHERBARROW \\ Manchester
}

\begin{abstract}
SUMMARY
Upper eyelid entropion is a complication of chronic blepharoconjunctivitis which may be easily missed unless careful examination of the lid margin of patients with trichiasis is carried out. Many patients undergo years of unsuccessful treatment for trichiasis because the underlying upper eyelid entropion has not been detected. We would like to recommend the already established procedure of anterior lamellar repositioning as a more permanent solution to this distressing condition and present the results of this procedure on 19 consecutive patients ( 28 lids). Our surgical technique is described and the results in this group of patients reported. The procedure was successful in 24 of 28 eyelids (85\%), with success being defined as complete resolution of symptoms for a follow-up period of at least 10 months. Anterior lamellar repositioning is easy and relatively quick to perform and provides good functional and cosmetic results.
\end{abstract}

The commonest cause of upper lid entropion worldwide is trachoma. In most UK ophthalmic departments, however, chronic blepharoconjunctivitis is the commonest cause of this condition. Other less frequent causes include Stevens-Johnson syndrome, mucous membrane pemphigoid, chemical trauma, prolonged use of topical glaucoma medication, postenucleation socket syndrome, herpes zoster ophthalmicus, vernal keratoconjunctivitis, dysthyroid eye disease and following ptosis surgery. A wide variety of procedures have been described to deal with this condition. ${ }^{1-4}$ Many patients with upper eyelid entropion are, however, inadequately labelled as having trichiasis, ${ }^{5}$ and make frequent visits to their eye department for various forms of treatment, all of which cause some discomfort and are time consuming for the doctor and patient, in addition to having a poor long-term success rate. The technique of

Correspondence to: B. Leatherbarrow, Manchester Royal Eye Hospital, Oxford Road, Manchester M13 9WH, UK. anterior lamellar reposition ${ }^{6}$ is a relatively simple procedure to perform and we have found it to have a good rate of success.

\section{METHODS}

The case notes of all patients with upper eyelid entropion secondary to chronic blepharoconjunctivitis who had the procedure of anterior lamellar reposition over a 3 year period were reviewed. The notes on a total of 19 patients on whom 28 anterior lamellar reposition procedures had been carried out were retrieved. The grade of entropion for each case was identified where available, based on the grading system described by Kemp and Collin. ${ }^{7}$ They define minimal entropion as posterior migration of the meibomian gland orifices, minimal or complete conjunctivalisation of the lid margin, and the lashes touching the globe on up-gaze. Moderate entropion is defined by lashes touching the globe in the primary position in addition to the above, with or without thickening of the tarsal plate. Severe upper lid entropion is defined by a totally disorganised margin with metaplastic lashes and trichiasis, definite lid retraction, and keratin formation in plaques on the palpebral conjunctival surface.

There was a history of previous treatment for trichiasis in all 28 lids. The treatments included electrolysis, cryotherapy and epilation, with previous lid splitting procedures having been carried out at other units on 2 lids $(7 \%)$. All cases fell into the minimum and moderate categories based on the above grading system.

\section{Surgical Procedure}

A skin crease incision was marked in the upper eyelid using gentian violet and a cocktail stick. A $1-1.5 \mathrm{ml} 50-50$ mixture of $0.5 \%$ marcaine with $1: 200$ 000 units of adrenaline and $2 \%$ lignocaine was injected subcutaneously into the upper eyelid. The patient was then prepared and draped in the usual 


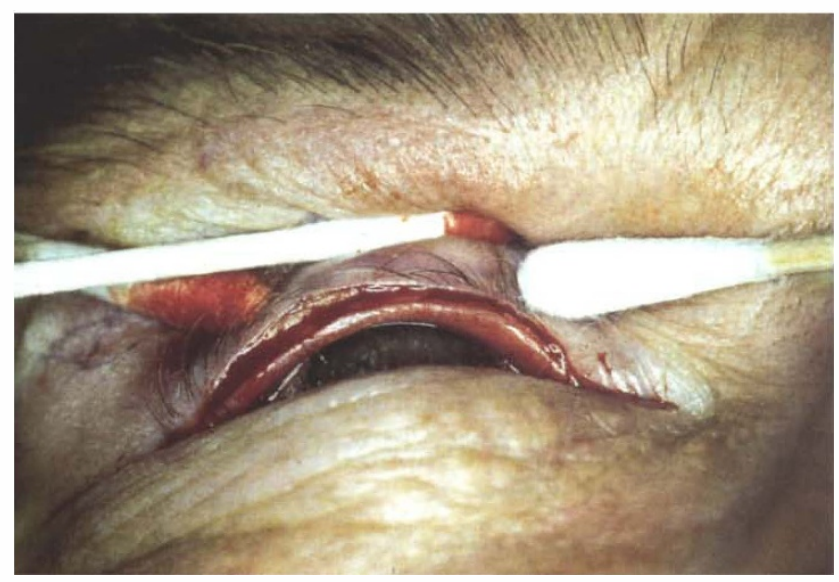

Fig. 1. Incision along the length of the upper eyelid margin through the grey line to a depth of at least $2 \mathrm{~mm}$, stopping short of the punctum.

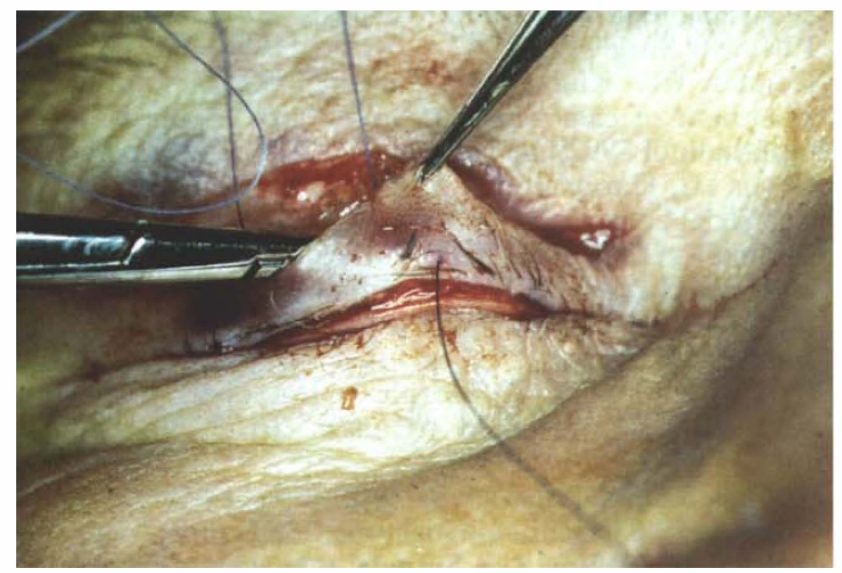

Fig. 3. The second arm of the 5.0 vicryl suture being passed through the lash line with the other limb already passed through and visible alongside.

sterile ophthalmic fashion. An incision to a depth of $2 \mathrm{~mm}$ was made through the grey line along the length of the upper eyelid margin using an MSP Super Sharps Blade no. 7513 (Fig. 1). Any oozing resulting from this incision was left to clot spontaneously and attention was now turned to the upper eyelid skin crease where an incision was made using a no. 15 Bard Parker blade. Using Paufique forceps on either side of the incision mark, Wescott scissors were used to dissect down through the orbicularis oculi muscle to the superior border of the tarsal plate. The skin and orbicularis muscle were dissected free from the anterior surface of the tarsal plate, stopping just short of the lash roots. A double-armed 5.0 vicryl suture was passed horizontally through the centre of the exposed tarsal plate $5 \mathrm{~mm}$ up from the lid margin (Fig. 2), both arms of the suture then being passed from posterior to anterior through the anterior lamella just above the lashes (Fig. 3). Two further sutures were placed in a similar fashion medial and lateral to the first suture. All three were

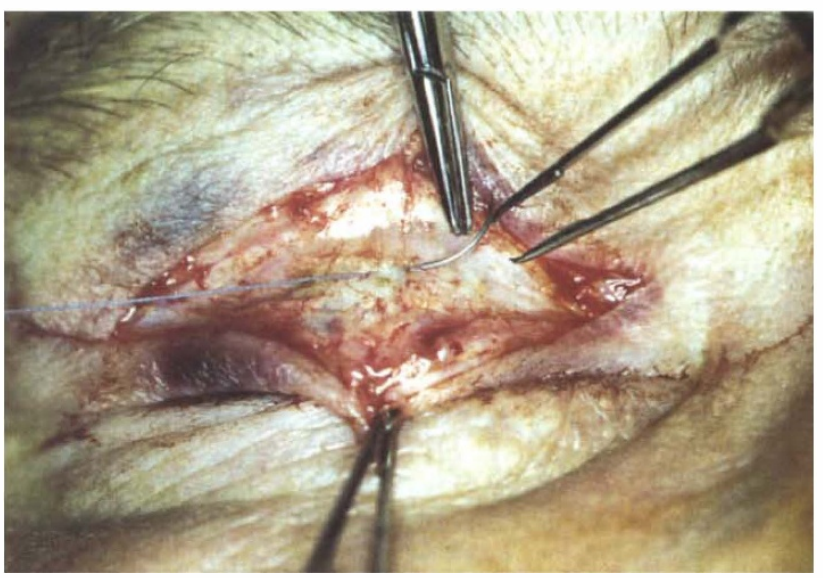

Fig. 2. Incision through the upper eyelid skin crease with the tarsal plate exposed. One arm of a double-armed 5.0 vicryl suture has been passed through the tarsal plate.

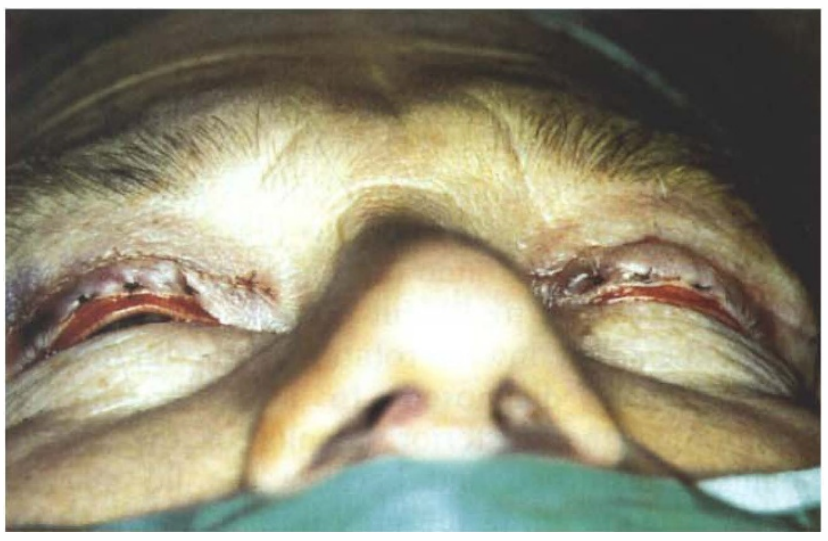

Fig. 4. Final position of the anterior lamellae showing three 5.0 vicryl sutures tied securely and the skin crease incision edge reapproximated with 7.0 vicryl incorporating the levator aponeurosis.

then tied securely, aiming for initial overcorrection, and the skin crease incision edges were reapproximated using 7.0 vicryl sutures incorporating the levator aponeurosis (Fig. 4). Topical antibiotic was applied to the wound.

\section{RESULTS}

The results were based on patient satisfaction in terms of resolution of symptoms and cosmetic appearance, and on clinical examination of the lid by the clinician, with a minimum follow-up period of 10 months (range 10-34 months; mean 16 months). There were no peroperative and no immediate postoperative complications. All patients were satisfied with the post-operative cosmetic appearance. Surgery on 24 eyelids $(85 \%)$ resulted in complete resolution of symptoms, with the 2 eyelids which had had previous surgery being included in this group. Clinical examination of all these lids was satisfactory with no lid malposition or trichiasis seen. In the case of 4 lids (14\%) symptoms remained 
following surgery, with occasional epilation being required to manage mild residual trichiasis (3 eyelids) and further surgery required in 1 eyelid which had recurrence of entropion. All 4 eyelids in the unsuccessful group had been graded preoperatively as moderate entropion; we were unable to identify the reasons for failure in this group but assume that the recommended initial overcorrection at surgery was insufficient.

\section{DISCUSSION}

Upper eyelid entropion secondary to chronic blepharoconjunctivitis can be easily and successfully treated by the well-described procedure of anterior lamellar reposition. Treatment of trichiasis with epilation, electrolysis or cryotherapy is tedious and in the presence of upper eyelid entropion will be unrewarding. The disease process in chronic blepharoconjunctivitis principally involves damage to the lid margin architecture. The resulting lid border entropion occurs because of a relative shortage of posterior lamellar tissue in comparison with anterior ${ }^{7}$ and is recognised clinically by conjunctivalisation of the meibomian gland orifices and anterior placement of the mucocutaneous junction. ${ }^{5}$ In our series of patients we found that many had had more than one previous treatment episode for trichiasis because the subtle inward rotation of the lid margin had not been diagnosed. In anterior lamellar repositioning the posterior lamella of the eyelid remains undisturbed and because the lid margin incision is made along the grey line, a sharp, clean edge to the posterior lamella can be readily achieved.
Procedures which involve a tarsotomy incision made parallel to the lid margin result in a less well defined lid margin and, therefore, a less satisfactory cosmetic result. All our patients fell into the mild or moderate category of entropion and were therefore well suited to this procedure. Patients with severe entropion would, however, require tarsal fracture and rotation of the distal fragment. ${ }^{2}$ Achieving high rates of success with the anterior lamellar reposition procedure relies on accurate grading of the entropion and confining use of the procedure to cases in the mild and moderate groups.

Key words: Anterior lamellar repositioning, Upper eyelid entropion.

\section{REFERENCES}

1. Wojono TH. Lid splitting with lash resection for cicatricial entropion and trichiasis. Ophthalmic Plast Reconstr Surg 1992;8:287-9.

2. Kersten RC, Kleiner FP, Kulwin DR. Tarsotomy for treatment of cicatricial entropion with trichiasis. Arch Ophthalmol 1992;110:714-7.

3. Baylis HI, Harnake C. Tarsal grafting for correction of cicatricial entropion. Ophthalmic Surg 1979;10:42-8.

4. Millman AL, Katzen LB, Putterman AM. Cicatricial entropion: an analysis of its treatment with transverse blepharotomy and marginal rotation. Ophthalmic Surg 1989;20:575-9.

5. Barber K, Dabbs T. Morphological observations on patients with presumed trichiasis. $\mathrm{Br} \mathrm{J}$ Ophthalmol 1988;72:17-22.

6. Collin JRO. A manual of systematic eyelid surgery. Edinburgh: Churchill-Livingstone, 1983.

7. Kemp EG, Collin JRO. Surgical management of upper lid entropion. Br J Ophthalmol 1986;70:575-9. 